\title{
SPECIFIC HEAT AND MAGNETORESISTANCE OF Y-, Tl- AND Bi-TYPE HIGH-TEMPERATURE SUPERCONDUCTORS
}

\author{
R. Zalecki, A. Kozlowski, A. KolodziejczyK \\ Department of Solid State Physics, University of Mining and Metallurgy \\ Al. Mickiewicza 30, 30-059 Kraków, Poland
}

G. Gritzner and M. Mair

Johannes Kepler University, Institut of Chemical Technology, 4040 Linz, Austria

Specific heat of polycrystalline $\mathrm{DyBa}_{2} \mathrm{Cu}_{3} \mathrm{O}_{7}$ and $\mathrm{Tl}_{0.58} \mathrm{~Pb}_{0.42} \mathrm{Sr}_{1.6} \mathrm{Ba}_{0.4} \mathrm{Ca}_{2} \mathrm{Cu}_{3} \mathrm{O}_{9}$ samples, as well as the single crystal of $\mathrm{Bi}_{2} \mathrm{Sr}_{2} \mathrm{CaCu}_{2} \mathrm{O}_{8}$ have been measured within the temperature interval from 50 to $250 \mathrm{~K}$. For Dy- and Tl-specimens the pronounced jump in specific heat and apparent contribution from Gaussian fluctuations of superconducting order parameter close to $T_{\mathrm{c}}$ have been observed. In contrary, for Bi-specimen only a rounded maximum within a broad interval around $T_{c}$ has been detected. Magnetoresistance measurements as a function of temperature just below $T_{c}$ have been carried out for $\mathrm{Dy}$ - and Tl-samples and the slopes of upper critical fields have been determined. The data have been analysed within a frame of Ginzburg-Landau-Abrikosov- Gorkov theory with additional Gaussian-like fluctuation term. The electronic specific heat coefficients $\gamma$, and the coherence length $\xi$ have been obtained.

PACS numbers: 74.72.Bk, 74.72.Fq, 74.72.Hs, 74.25.Ha, 74.25.Bt

The specific heat anomaly, especially the specific heat jump $\Delta C$ at the superconducting transition temperature $T_{\mathrm{c}}$ in high-temperature superconductors (HTSC) of yttrium, thalium and bismuth families have been thorougly investigated in view to look at the discrepancy between BCS-like and fluctuations-added-like behavior [1-3]. The classical BCS-Ginzburg-Landau (BCS-GL) approach [4] neglects any fluctuations because according to the so-called Ginzburg criterion $[4,5]$,

$$
\frac{\left|T-T_{\mathrm{c}}\right|}{T_{\mathrm{c}}}<\left[\frac{k T_{\mathrm{c}}}{H_{\mathrm{c}}^{2}(0) \xi^{3}(0)}\right]^{2}=1.07 \times 10^{-9} \frac{\kappa_{G L}^{4} T_{c}^{2}}{H_{c 2}(0)}
$$

the temperature interval around $T_{\mathrm{c}}$, in which this theory collapses, is extremally narrow for conventional superconductors. In Eq. (1) $T_{\mathrm{c}}, H_{\mathrm{c}}, \xi$ have their usual meaning, $H_{\mathrm{c}}(T)=H_{\mathrm{c} 2}(T) 2^{-1 / 2} \kappa_{\mathrm{GL}}^{-1}$, and the so-called G-L parameter $\kappa_{\mathrm{GL}}$ is the ratio of the penetration depth $\lambda_{\mathrm{GL}}$ to the coherence length $\xi_{\mathrm{GL}}$. On the contrary, for the HTSC, the temperature interval is of the order of $0.1-1 \mathrm{~K}[5,6]$, mainly due 
to a very short coherence length, and the fluctuations may play a predominant role in a wide temperature region close to $T_{\mathrm{c}}$. The fluctuations are usually modelled as a Gaussian like contribution to the specific heat above and below $T_{\mathrm{c}}$ given by the following expression $[4,7]$ :

$$
\Delta C_{f}=C_{ \pm} t^{-(2-d / 2)}
$$

with $t=\left|T / T_{\mathrm{c}}-1\right|, C_{+}=k_{\mathrm{B}}\left[8 \pi \xi_{\mathrm{GL}}^{3}(0)\right]^{-1}$, and $C_{+} / C_{-}=n\left(2^{d / 2}\right)^{-1}$, where $d$ is the dimensionality and $n$ is the number of components of the order parameter.

In this paper we have focused attention on analysis of our heat capacity data of $\mathrm{DyBa}_{2} \mathrm{Cu}_{3} \mathrm{O}_{7}$ [6] (DyBCO), $\mathrm{Tl}_{0.58} \mathrm{~Pb}_{0.42} \mathrm{Sr}_{1.6} \mathrm{Ba}_{0.4} \mathrm{Ca}_{2} \mathrm{Cu}_{3} \mathrm{O}_{9}$ (TlBCCO) and $\mathrm{Bi}_{2} \mathrm{Sr}_{2} \mathrm{CaCu}_{2} \mathrm{O}_{8}$ (BiSCO) specimens in the vicinity of $T_{\mathrm{c}}$ in order to calculate properly the electronic specific heat coefficients $\gamma$. Some other macroscopic normaland superconducting-state quantities have been calculated taking into account our experimental data on the slopes of $\mathrm{d} H_{\mathrm{c} 2} /\left.\mathrm{d} T\right|_{T_{\mathrm{c}}}$.

Polycrystalline DyBCO and TIBCCO samples were prepared by the standard sintering procedure and sol-gel liquid-mix sintering technique, respectively [8]. A single crystal of BiSCO has been grown by travelling solvent floating zone method. Specific heat of all samples was measured by the adiabatic technique (and the continuous heating technique for $\mathrm{DyBCO}$ ) within the temperature range from 50 to $250 \mathrm{~K}$ in the arrangement described elsewhere [9]. The results of the measurements are presented in Fig. 1a. Resistivity was measured as a function of applied magnetic field and temperature by the standard ac resistance method. The temperature dependence of $H_{\mathrm{c} 2}$ has been calculated for the given magnetic field and the relevant zero-resistance temperature; this is shown in the inset of Fig. 1b for Dy-specimen, and in Fig. 2 for Tl-specimen.

a)

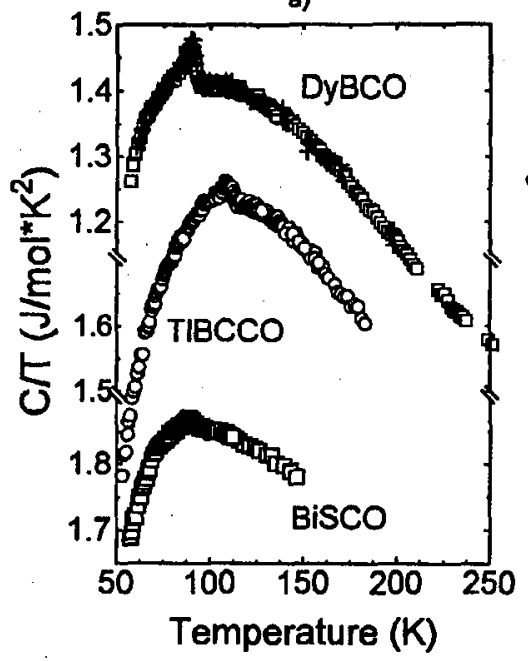

b)

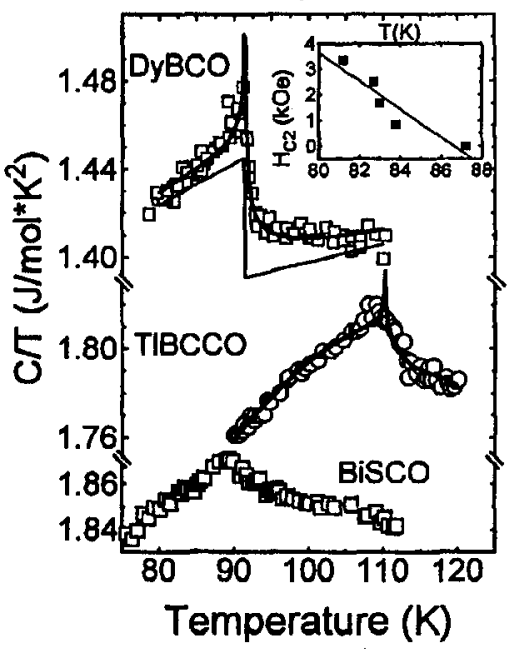

Fig. 1. $C / T$ vs. $T$ plots for investigated samples, where $C$ is the measured specific heat; (a) full plots, (b) $T$-region close to $T_{c}$ together with the fitting to the model (Eq. (3)), the inset: the upper critical field $H_{\mathrm{c} 2}(T)$ with its slope (solid line) for Dy-specimen. 


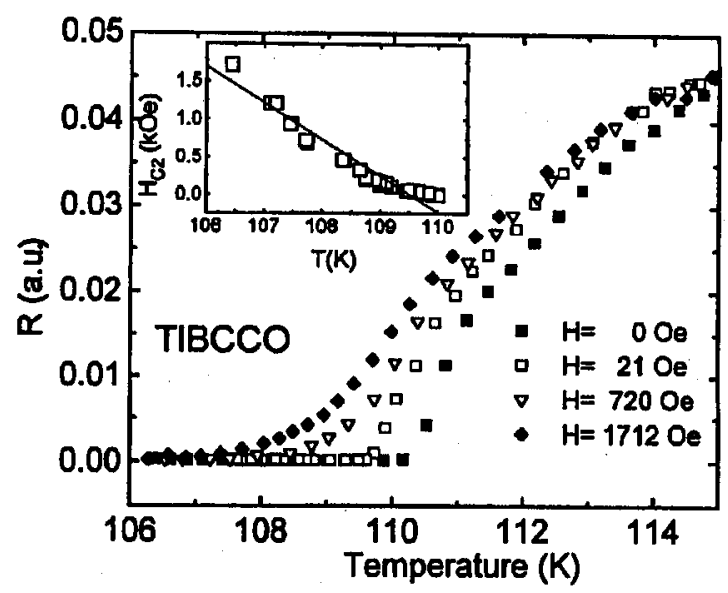

Fig. 2. Magnetoresistance data together with the temperature dependence of upper critical field $H_{c 2}$ and its slope (solid line) for Tl-specimen (inset).

Due to a possibly large fluctuation component to specific heat jump at $T_{c}$, we have tried to fit our data, close to $T_{\mathrm{c}}$ to the relation

$$
\frac{C}{T}=a+b t+c t^{2}+1.43 \gamma(1+1.83 t)+\frac{\Delta C_{f}}{T} .
$$

The term $a+b t+c t^{2}$ accounts for both lattice specific heat and any slowly varying specific heat component (e.g. magnetic contribution from either $\mathrm{Dy}^{+3}$ ions or antiferromagnetic correlations of $\mathrm{Cu}^{+2}$ sublattice) in a region close to the transition. The component $1.43 \gamma(1+1.83 t)$ describes BCS specific heat excess above the normal electronic component in the vicinity of $T_{\mathrm{c}}[10]$. The last term describes Gaussian fluctuations $[4,10]$. The results of fitting for Dy and Tl samples are shown in Fig. 1b, where also BCS contribution to the peak for Dy sample is shown. For DyBCO a satisfactory fit was obtained with a linear lattice component (i.e. $c=0$ ), while Tl-sample required a full quadratic lattice form. For both samples the best results were obtained for $d=3$. As is apparent from Fig. 1b the quality of fitting is better for DyBCO than for TIBCCO. This type of analysis fails completely for BiSCO, because only a rounded maximum within a broad temperature interval around $T_{\mathrm{c}}$ has been detected. The specific heat jumps at $T_{\mathrm{c}}, \gamma$ coefficients as calculated from fitting procedures described above, $\mathrm{d} H_{\mathrm{c} 2} /\left.\mathrm{d} T\right|_{T_{\mathrm{c}}}$ from magnetoresistance data of Fig. 1b and Fig. 2 and G-L coherence length $\xi_{\text {GL }}$ as calculated from Eq. (2) for $C_{+}$, are collected in Table.

The coefficient $\gamma$ can be also calculated from $\mathrm{d} H_{\mathrm{c} 2} /\left.\mathrm{d} T\right|_{T_{c}}$ based on the GLAG formulae in the dirty limit of superconductivity, most likely valid also for HTSC,

$$
\left.\frac{\mathrm{d} H_{\mathrm{c} 2}}{\mathrm{~d} T}\right|_{T_{\mathrm{c}}}=4.48 \gamma \rho
$$

where $\gamma$ is in $\left[\mathrm{erg} / \mathrm{cm}^{3} \mathrm{~K}^{2}\right.$ ] and $\rho$ is normal state resistivity just above $T_{c}$ in $[\Omega \mathrm{cm}]$. 
TABLE

Experimental $T_{\mathrm{c}}, \Delta C / T_{\mathrm{c}}, \mathrm{d} H_{\mathrm{c} 2} /\left.\mathrm{d} T\right|_{T_{\mathrm{c}}}$ and calculated $\gamma, \xi_{\mathrm{GL}}$ values for investigated samples.

\begin{tabular}{c|c|c|c|c|c}
\hline \hline & $T_{\mathrm{c}}(\mathrm{K})$ & $\begin{array}{c}\Delta C / T_{\mathrm{c}} \\
{[\mathrm{mJ} /(\mathrm{mol} \mathrm{K})]}\end{array}$ & $\begin{array}{c}\mathrm{d} H_{\mathrm{c} 2} /\left.\mathrm{d} T\right|_{T_{\mathrm{c}}} \\
{[\mathrm{kOe} / \mathrm{K}]}\end{array}$ & $\begin{array}{c}\gamma \\
{\left[\mathrm{mJ} /\left(\mathrm{mol} \mathrm{K}^{2}\right)\right]}\end{array}$ & $\xi_{\mathrm{GL}}[\AA]$ \\
\hline DyBCO & $91.4 \pm 0.1$ & $53 \pm 1$ & $-0.55 \pm 0.03$ & $38.2 \pm 0.5$ & $6.5 \pm 0.5$ \\
TlBCCO & $110.0 \pm 0.2$ & $30 \pm 2$ & $-0.48 \pm 0.03$ & $25.9 \pm 0.5$ & $5.0 \pm 0.5$ \\
BiSCO & $87 \pm 0.5$ & $19 \pm 4$ & - & - & -
\end{tabular}

The $\gamma$ values calculated in such way are comparable to the respective values from Table, but are systematically smaller.

The main conclusion from the experimental and calculated superconducting parameters $\Delta C, \mathrm{~d} H_{\mathrm{c} 2} /\left.\mathrm{d} T\right|_{T_{\mathrm{c}}}, \xi_{\mathrm{GL}}$ and normal-state $\gamma^{\prime}$ 's is that these values systematically decrease in the sequence depicted in Table (from Dy-to Bi-specimens). If it is intrinsic property of the specimens (i.e. does not depend on the preparation procedure, e.g. oxygen content and microstructure) then it might support the theoretical suggestion that the specific heat jump at $T_{\mathrm{c}}$ decreases due to increasing anisotropy of the superconducting properties from YBCO to BiSCO type HTSC [11]. Further experimental data and more discussion on the subject will be published elsewhere.

Some of us (A.K., A. K. and R. Z.) gratefully acknowledge support from the Committee for Scientific Research.

\section{References}

[1] W. Schnelle, E. Braun, H. Broicher, H. Weiss, H. Geus, S. Ruppel, M. Galffy, W. Braunisch, A. Waldorf, F. Seidler, D. Wohlleben, Physica C 161, 123 (1989).

[2] W. Schnelle, E. Braun, H. Broicher, P. Domel, S. Ruppel, W. Braunisch, J. Harnischmacher, D. Wohlleben, Physica $C$ 168, 465 (1990).

[3] A.I. Sokolov, Physica C 174, 208 (1991).

[4] A. Junod, in: Physical Properties of HTSC II, Ed. D.M. Ginsberg, World Scientific, Singapore 1990, p. 13.

[5] C.J. Lobb, Phys. Rev. B 36, 3930 (1987).

[6] A. Kozłowski, Z. Tarnawski, A. Kołodziejczyk, J. Chmist, T. Ściężor, R. Zalecki, Physica C 184, 113 (1991).

[7] S.K. Ma, Modern Theory of Critical Phenomena, Benjamin, Reading (MA) 1976.

[8] J. Chmist, Ph.D. Thesis, University of Mining and Metallurgy, Cracow 1991 (in Polish).

[9] Z. Tarnawski, Ph.D. Thesis, University of Mining and Metallurgy, Cracow 1986 (in Polish).

[10] S.E. Inderhees, M.B. Salamon, Nigel Goldenfeld, J.P. Rice, B.G. Pazol, D.M. Ginsberg, J.Z. Liu, G.W. Crabtree, Phys. Rev. Lett. 60, 1178 (1988).

[11] T. Schneider, M. Frick, in: Strong Correlation and Superconductivity, Eds. H. Fukuyama, S. Maekawa, A.P. Malozemoff, Springer Series in Solid State Sciences, Vol. 89, Springer Verlag, Berlin 1989, p. 176. 